\title{
Limitações no desempenho ocupacional de indiví- duos portadores de hemofilia em Centro Regional de Hemoterapia de Ribeirão Preto, Brasil ${ }^{*}$
}

\section{Limitations in occupational performance of indi- viduals with hemophilia in Hemotherapy Regional Center of Ribeirão Preto, Brazil}

\author{
Danielle Aline Barata-Assad ${ }^{1}$, Valéria Meirelles Carril Elui ${ }^{2}$
}

\begin{abstract}
BARATA-ASSAD, D. A.; ELUI, V. M. C. Limitações no desempenho ocupacional de indivíduos portadores de hemofilia em Centro Regional de Hemoterapia de Ribeirão Preto, Brasil. Rev. Ter. Ocup. Univ. São Paulo, v. 21, n. 3, p. 198-206, set./dez. 2010.
\end{abstract}

\begin{abstract}
RESUMO: A hemofilia é grave distúrbio hereditário da coagulação sanguínea que resulta em incapacidades que influenciam no desempenho dos acometidos. A tecnologia assistiva (TA) é equipamento ou conjunto de produtos, comprados ou modificados ou feitos sob medida, usados ampliar o desempenho funcional. Esta pesquisa teve como objetivo identificar as limitações no desempenho ocupacional dos hemofílicos e verificar a influência dos recursos de TA na funcionalidade destes. O estudo foi realizado de janeiro a julho de 2009 no Centro Regional de Hemoterapia do HCFMRPUSP. Todos os indivíduos portadores de hemofilia em seguimento foram convidados. Participaram 42 indivíduos através da aplicação de roteiros para o levantamento dos dados sócio-demográficos e clínicos; das barreiras e facilitadores da acessibilidade na residência e na comunidade; dos recursos da TA utilizados nas Atividades de Vida Diária (AVD's), na mobilidade em sua residência e na comunidade e o instrumento de avaliação da funcionalidade HAQ (Health Assessment Questionnaire).Os resultados demonstram que a maioria dos entrevistados apresentam limitação nas AVD's, Atividades Produtivas e de Trabalho, mobilidade; e obtiveram classificação "com alguma dificuldade" no HAQ. Os recursos de TA utilizados pela maioria dos participantes estavam relacionados à higiene e uso do vaso sanitário e dispositivos de ajuda para deambulação.
\end{abstract}

DESCRITORES: Terapia ocupacional; Hemofilia A; Equipamentos de auto-ajuda/utilização.

\footnotetext{
*Artigo baseado no Trabalho de Conclusão de curso apresentado ao Curso de Terapia Ocupacional da Faculdade de Medicina de Ribeirão Preto - Universidade de São Paulo, realizada com apoio da Fundação de Amparo à Pesquisa do Estado de São Paulo - FAPESP, intitulado: Terapia Ocupacional, Hemofilia e Tecnologia Assistiva. Ribeirão Preto, 2009

1. Terapeuta Ocupacional - Faculdade de Medicina de Ribeirão Preto - Universidade de São Paulo. Bolsista de Iniciação Científica da Fundação de Amparo à Pesquisa do Estado de São Paulo - FAPESP.

2. Docente do curso de Terapia Ocupacional da Faculdade de Medicina de Ribeirão Preto - Universidade de São Paulo.

Endereço para correspondência: Rua das Paineras, Casa 2, Campus USP- Ribeirão Preto, SP CEP:14031-390 e-mail:velui@fmrp.usp.br
} 


\section{INTRODUÇÃO}

s hemofilias são coagulopatias hereditárias,
exemplo clássico de doenças recessivas
ligadas ao cromossomo X, na qual a deficiência quantitativa ou defeitos moleculares dos fatores de coagulação VIII e IX, respectivamente, hemofilia A e $\mathrm{B}$, causam dificuldade na formação de fibrina, resultando em hemorragia. São classificadas de acordo com os níveis plasmáticos do fator deficiente circulante em leve (com FVIII e IX entre 5 e $25 \%$ ou 5 e $25 \mathrm{U} / \mathrm{dL}$ ), moderada (com FVIII e IX entre 1 e $5 \%$ ou 1 a $5 \mathrm{U} / \mathrm{dL}$ ) e grave (FVIII e IX inferior a $1 \%$ ou $1 \mathrm{U} / \mathrm{dL}$ ) (ANTUNES, 2007).

A forma leve da hemofilia é caracterizada por sangramentos pouco freqüentes, desencadeados por traumas (acidentes, procedimentos cirúrgicos, extrações dentárias, etc.) Na classificação moderada, os hematomas e hemartroses são menos freqüentes que na forma grave e nem sempre estão associados a traumatismos evidentes. Mas, é necessário realizar o tratamento adequado como forma de prevenção da evolução desses sintomas para sangramentos importantes e freqüentes, e conseqüentemente a expressão da doença mais grave do que o esperado (ZAGO et al., 2001).

$\mathrm{Na}$ forma grave da doença há sangramentos de repetição ( 2 a 4 por mês) e hemartroses graves, as quais, quando não tratadas adequadamente, evoluem para artropatias crônicas e incapacitantes. As hemorragias são intermitentes e alguns pacientes podem ficar semanas ou meses sem apresentar manifestações hemorrágicas (ZAGO et al. 2001).

Assim, "a hemofilia grave e moderada são as que mais causam deformidades e limitações às condições de vida do doente, levando por vezes, a incapacidades permanentes e seqüelas irreversíveis que influenciam no modo de vida da pessoa" (SANTOS et al., 2007, p. 87).

As hemartroses e os sangramentos musculares são as manifestações hemorrágicas mais comuns e mais debilitantes nos hemofílicos, que acometem principalmente as articulações dos joelhos, cotovelos, tornozelos, ombro, coxo-femorais e punhos (ZAGO et al., 2001).

É caracterizada por sensação de calor e formigamento nas primeiras duas horas, posteriormente, há leve desconforto e restrição de movimento, seguida de horas de dor, aumento de volume e de temperatura e limitação da mobilidade articular. Após tratamento adequado, há melhora da dor e diminuição da sensação de pressão intra-articular (CARAPEBA, 2006).

As articulações acometidas pela hemartrose retornam ao estado funcional normal, porém, a recorrência dos sangramentos predispõe a articulação á sangramentos secundários a traumatismos mínimos. Com isso, há redução do volume articular devido ao enfraquecimento das estruturas periarticulares e ao acometimento das estruturas ósseas adjacentes, e conseqüente diminuição de sua mobilidade (ZAGO et al., 2001; LORENZI, 2003).

O estágio final da hemartrose é a artropatia hemofílica crônica, que é caracterizada por perda da movimentação articular devido às contraturas fixas em flexão e intensa atrofia muscular, secundária ao desuso; além de deformidades da extremidade afetada (VILLAÇA et al., 2001).

Os hematomas musculares podem ocorrer espontaneamente ou após traumatismos mínimos. Oferecem risco quando aumentam progressivamente, podendo comprimir estruturas nobres. Ocorrem mais na panturrilha (podendo causar paralisia de nervos e/ou deformidade), coxa, glúteos e antebraço (onde podem causar paralisia dos nervos mediano ou ulnar ou a contratura isquêmica da mão, a síndrome de Volkmann) e no músculo ileopsoas, local critico para desenvolvimento de cistos e tumores (VILLAÇA et al., 2001; ANTUNES, 2007).

A alta freqüência de episódios de hemorragias musculares e articulares poderá causar aos indivíduos portadores de hemofilia deformidades nos: joelhos, quadris e cotovelos em flexão; curvatura exagerada das costas (lordose lombar); flexão plantar dos tornozelos; assimetria pélvica devido à diferença de comprimento entre as pernas, além de intensidade variada de atrofia muscular. Há também a apresentação dos punhos e dedos flexionados devido à hemorragia muscular profunda no antebraço, que mesmo sendo mais incomuns, são extremamente incapacitantes (WFH, 2006).

Além dessas manifestações clínicas, podem ocorrer também o acometimento dos sistemas gastrointestinal, geniturinário, sistema nervoso central, mucosas e pele (ZAGO, 2001; ANTUNES, 2007).

A modalidade mais comum de tratamento da hemofilia no Brasil é o tratamento de demanda, ou seja, infusão de fator VIII ou IX na vigência de hemorragia. Há a profilaxia primária, na qual há reposição do fator deficiente de duas a três vezes por semana, desde o início da infância (entre dois e três anos de idade), antes dos sangramentos começarem, sendo eficaz na prevenção da artropatia crônica hemofílica. No entanto, esse tratamento é de alto custo. E, a profilaxia secundária, que consiste em infusões regulares do fator deficiente após ter sido identificada uma articulação proeminente a sangramentos freqüentes, com o objetivo de limitar as hemorragias e a lesão articular (CARAPEBA, 2006).

A hemofilia é, portanto, doença crônica caracterizada pelo acometimento do sistema músculo - esquelético que gera a restrição da mobilidade articular e o desenvolvimento de deformidades musculares, podendo assim, limitar a funcionalidade destes indivíduos, nas áreas e contextos 
de desempenho.

Por isso, o terapeuta ocupacional (TO), com sua visão integrada do ser humano com o foco principal na ocupação humana, ou seja, a atividade e participação social e através do trabalho de reabilitação poderá auxiliar os indivíduos acometidos pela hemofilia a explorar seus potenciais funcionais máximos, restaurando sua função, habilitando-os e reabilitando-os.

Para isso, o TO poderá utilizar os recursos da tecnologia assistiva como meio de adaptação terapêutica, dispositivo de auxílio ou equipamento de auto-ajuda, incluindo no processo terapêutico o planejamento ou a modificação estrutural de um ambiente físico como forma de facilitar o desempenho de atividades de autocuidado, trabalho e lazer. (LUZO et al., 2004).

Diante disso, o presente estudo tem como propósito conhecer quais são as limitações no desempenho ocupacional dos indivíduos portadores de hemofilia que recebem tratamento no Centro Regional de Hemoterapia do Hospital das Clínicas da Faculdade de Medicina de Ribeirão Preto da Universidade de São Paulo (CRH-FMRP-USP), verificar qual é a influência da tecnologia assistiva para a funcionalidade destes.

\section{METODOLOGIA}

Trata-se de um estudo descritivo quantitativo, realizado no CRH-HCFMRP-USP-SP, no período de janeiro a julho de 2009, com a devida autorização do Comitê de Ética em Pesquisa do HCFMRP-USP, em sua 277", Reunião Ordinária realizada em 24/11/2008, bem como o Termo de Consentimento Livre e Esclarecido, de acordo com o Processo HCRP n ${ }^{\circ}$. 9714/2008.

Na coleta de dados foram utilizados os instrumentos elaborados pelas próprias pesquisadoras, de acordo com os preceitos da Classificação Internacional da Funcionalidade (CIF), para delimitar quais as funções e estruturas do corpo apresentam integridade ou deficiência; conhecer o quanto a atividade está sendo realizada ou não pelo indivíduo no seu dia-dia; avaliar se a participação está preservada ou restrita; e assim, diagnosticar os fatores ambientais que facilitam ou dificultam sua execução e a participação dos indivíduos portadores de hemofilia, visando conhecer assim a sua funcionalidade.

Os roteiros que serão explicitados abaixo foram elaborados pelos autores.

$O$ roteiro para levantamento dos dados sóciodemográficos com 12 questões foi utilizado para coletar informações tais como: a idade, escolaridade, procedência, estado civil, profissão e situação profissional atual.

O roteiro para levantamento dos dados clínicos com 25 questões foi utilizado para coletar informações tais como: tipo de hemofilia, classificação, co-morbidades associadas, tratamento, utilizado e articulações acometidas pelas manifestações hemorrágicas.

$O$ roteiro para levantamento das barreiras e facilitadores da acessibilidade e da mobilidade do paciente em sua residência e na comunidade, composto por 8 questões, foi utilizado para identificar as dificuldades de desempenho dos indivíduos na execução das Atividades de Vida Diária (AVD's), por exemplo: alimentar-se, tomar banho, escovar os dentes etc.; nas Atividades de Trabalho e Produtivas (AVP's), exemplos: dirigir, escrever, fazer compras, desempenhar sua função profissional, etc.; nas Atividades de Lazer, exemplos: passear, visitar parentes, viajar, etc.; e mobilidade na casa e na comunidade. Para a graduação da dificuldade apresentada em cada área de desempenho foi utilizada a seguinte a escala de Liket, graduando-se nenhuma (0), pouca (1), média (2) e muita (3).

O roteiro para o levantamento dos recursos da tecnologia assistiva, composto por 7 questões, com nomes e fotos dos itens de TA, foi utilizado para auxiliar a identificação pelos indivíduos dos recursos utilizados nas AVD's, para alimentação (exemplos: talher engrossado, borda para prato, tábua com pregos, abridor elétrico, etc.), para vestuário (exemplos: calçador de meias, abotoador, puxador de zíper, etc.) para higiene pessoal (exemplos: esponja de cabo longo, sabonete amarrado, cadeira de banho, etc.) e para a mobilidade destes em sua residência e na comunidade (exemplo: bengala, muleta, cadeira de rodas, etc.).

O instrumento de avaliação de funcionalidade $\mathbf{H e a l}$ th Assessment Questionnaire (HAQ), traduzido, adaptado e validado para uso no Brasil, consiste em 20 questões sobre as AVD's (exemplos: vestir-se, inclusive amarrar os cordões dos sapatos e abotoar suas roupas; lavar sua cabeça e seus cabelos; levantar-se de maneira ereta de uma cadeira de encosto reto e sem braço, etc.) que são pontuadas conforme a capacidade de realizá-las: sem dificuldade (0), com alguma dificuldade (1), com muita dificuldade (2) e incapaz de fazer (3). As questões são divididas em oito domínios (vestir-se, levantar-se, alimentar-se, caminhar, higiene pessoal, alcançar objetos, apreender objetos e outras atividades); para a quantificação, é feita a média aritmética apenas do maior escore de cada componente. Os pontos variam de 0 a 3. Quanto menor a pontuação, melhor a capacidade funcional (FERRAZ et al., 1990). 
Ao final, os indivíduos foram questionados, através de uma pergunta aberta, sobre quais as dificuldades que este encontra no seu cotidiano e o que ele acredita que poderia ajudá-lo a superar estas dificuldades.

A amostra foi definida através do levantamento de dados dos prontuários dos indivíduos acometidos pela hemofilia cadastrados no CRH-FMRP-USP, que realizavam o tratamento multiprofissional na instituição, e não estavam em tratamento com a terapia ocupacional, pois não há este profissional contratado no Centro Regional, sendo os indivíduos encaminhados quando necessário para a TO do centro de Reabilitação do HCFMRP-USP.

Todos os indivíduos, maiores de 18 anos, de ambos os sexos, sem distinção de classe econômica ou escolaridade, que apresentassem o diagnóstico de hemofilia, nas formas moderadas e graves e que apresentassem capacidade para responder ao questionário e comparecer ao referido Centro Regional foram incluídos neste estudo.

Foram excluídos os casos de hemofilia leve e menores de 18 anos, aqueles que não apresentavam condições de responder ao questionário por problemas cognitivos, aqueles que já haviam falecido e aqueles que não podiam comparecer ao Centro de Hemoterapia neste período de 6 meses de pesquisa.

A partir disso, o número de prontuários que foram incluídos no estudo totalizou 101, sendo encaminhada carta convite a todos e agendado horário para realização da pesquisa no mesmo dia do retorno médico e, quando não houve o comparecimento, foi realizado contato telefônico.

Compareceram ao referido Centro no período de janeiro a julho de 2009, 47 indivíduos para o retorno médico e a profilaxia secundária, ou seja, as infusões regulares do fator deficiente após a identificação da articulação proeminente a sangramentos freqüentes. Isso possibilitou a coleta de dados para a pesquisa junto a 42 pacientes para o projeto piloto com 5 indivíduos.

O projeto piloto foi realizado com objetivo de adequar os instrumentos da pesquisa e para treinamento da pesquisadora na aplicação destes, sendo os dados coletados excluídos do estudo final.

Após a coleta, foi elaborado um banco de dados, utilizando o sistema operacional Windows XP pelo programa Microsoft Office Excel 2003 para posterior análise estatística, com o apoio do Centro de Métodos Quantitativos - HCFMRP-USP (CEMEC). As respostas à pergunta aberta foram transcritas pela pesquisadora utilizando-se do programa Microsoft Office Word 2003 e agrupadas de acordo com a similaridade de fatos relatados.

Foram analisadas e descritas, as freqüências em porcentagem (\%) através da estatística descritiva: os dados sócio-demográficos e clínicos, as barreiras arquitetônicas que dificultavam a acessibilidade e a mobilidade destes indivíduos em suas residências e na comunidade e os recursos da tecnologia assistiva utilizados. Foi analisado e quantificado segundo a validação estabelecida o escore de funcionalidade de acordo com a pontuação do HAQ.

Para verificar possíveis associações com variáveis de interesse, foi utilizado o teste Exato de Fisher. Para tais análises, foi utilizado o software SAS versão $9 \circledR$. Por exemplo, foi realizada a correlação dos dados obtidos, quanto a gravidade da doença, as deformidades apresentadas e as tecnologias assistivas utilizadas.

As respostas da pergunta aberta foram analisadas segundo similaridade de fatos, e utilizada somente para reforçar ou não as respostas sobre o uso das tecnologias e sobre como o próprio indivíduo percebe as potencialidades e possíveis ajudas que a tecnologia pode oferecer e, até apontar os caminhos, que cada um tomou para minimizar as incapacidades causadas pela hemofilia.

\section{RESULTADOS}

A análise dos dados encontrados possibilitou caracterizar a população estudada de acordo com o sexo, a faixa etária, tipo de coagulopatia, classificação da severidade e quais as articulações mais acometidas pelas hemartroses e artropatia hemofílica crônica.

A amostra foi composta por $100 \%$ de indivíduos portadores de hemofilia do sexo masculino, $38 \%$ encontramse na faixa etária de 18 a 25 anos, $28,5 \%$ encontram-se na faixa etária de 26 a 35 anos, $21,5 \%$ encontram-se na faixa etária de 36 a 45 anos e $12 \%$ encontram-se na faixa etária de 46 a 55 anos.

Dos entrevistados, $81 \%$ apresentavam hemofilia tipo A e $19 \%$ apresentava tipo B. Daqueles com hemofilia tipo A, $73 \%$ estavam classificados como grave e $27 \%$ como moderada. Entre aqueles com hemofilia tipo B, 62,5\% estavam classificados como grave e $37,5 \%$ como moderada.

Conforme Gráfico 1, as articulações mais acometidas pelas manifestações clínicas em ordem de maior freqüência, foram o joelho, o cotovelo e tornozelo.

A análise das limitações apresentadas nas áreas de desempenho e na mobilidade possibilitou conhecer quantos indivíduos apresentavam dificuldade na realização das AVD's, nas AVP's, Lazer e na mobilidade, conforme demonstra a Tabela 1. 
BARATA-ASSAD, D. A.; ELUI, V. M. C. Limitações. Rev. Ter. Ocup. Univ. São Paulo, v. 21, n. 3, p. 198-206, set./dez. 2010.

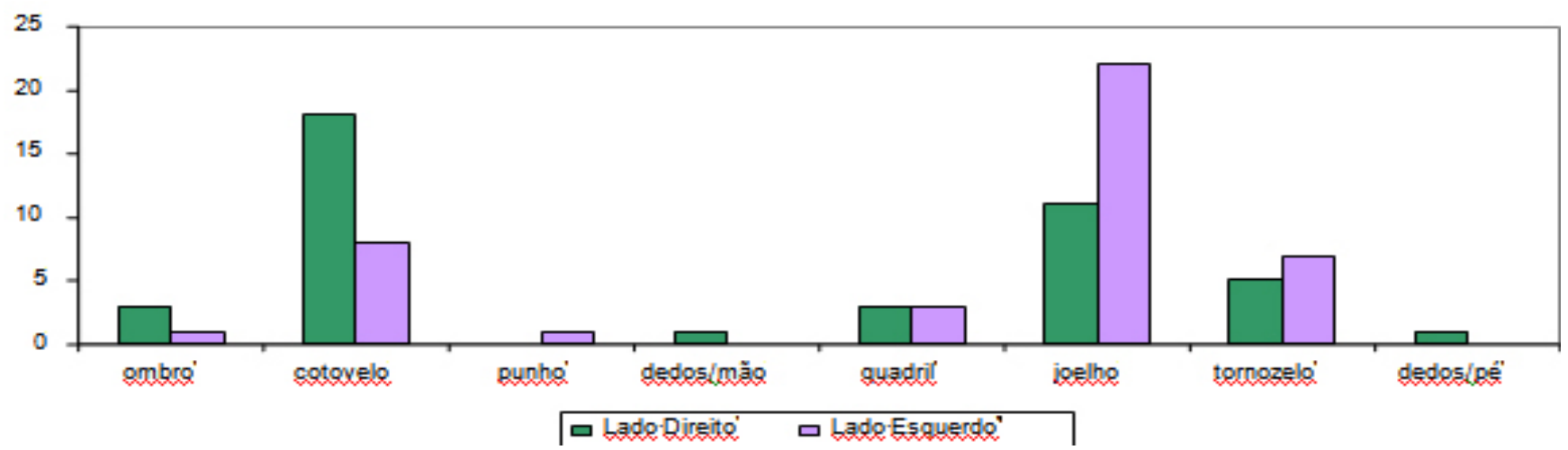

Gráfico 1 - Articulações mais cometidas pelas manifestações clínicas

Tabela 1 - Desempenho Ocupacional, segundo tipo e grau de dificuldade

\begin{tabular}{cccc}
\hline & Desempenho Ocupacional & & \\
\hline & Com dificuldade (n) & Sem Dificuldade (n) & Total \\
Atividades de Vida Diária & 19 & 23 & 42 \\
Atividades de Trabalho e Produtivas & 24 & 18 & 42 \\
Lazer & 13 & 29 & 42 \\
Mobilidade & 13 & 29 & 42 \\
\hline
\end{tabular}

Dentre as áreas de desempenho analisadas, a higiene no vaso sanitário mostrou-se de maior dificuldade para o desempenho dos indivíduos entrevistados, tanto que as tecnologias assistivas utilizadas por estes eram, em sua maioria, para esta área de desempenho, conforme a Tabela 2.

Tabela 2 - Tecnologias Assistivas utilizadas pelos indivíduos portadores de hemofilia

\begin{tabular}{lc}
\hline Tecnologias Assistivas & $\begin{array}{c}\text { Indivíduos que a } \\
\text { utilizam }\end{array}$ \\
\hline Cadeira de banho & 4 \\
Cadeira para sanitário & 4 \\
Esponja de cabo longo & 2 \\
Adaptação para fio dental & 1 \\
Adaptação no barbeador & 1 \\
Cadarços elásticos & 1 \\
Elevação no vaso sanitário & 2 \\
\hline Total & 15 \\
\hline
\end{tabular}

AAVP de maior comprometimento de desempenho foi a atividade profissional, tanto que dos entrevistados, 25 se encontravam fora do mercado de trabalho, enquanto que 19 estavam trabalhando.

Quanto à mobilidade, a grande maioria apresentava dificuldade de deambulação e utilizava recursos assistivos para mobilidade, conforme descrito na Tabela 3.

Tabela 3 - Distribuição dos dispositivos de ajuda para mobilidade utilizados

\section{Dispositivos de ajuda utilizados para a mobilidade}

\begin{tabular}{ccc}
\hline Dispositivos de ajuda & Indivíduos (n) & Porcentagem (\%) \\
Bengala & 5 & 11,9 \\
Muleta & 9 & 21,4 \\
Andador & 3 & 7,1 \\
$\begin{array}{c}\text { Cadeira de Rodas } \\
\text { Manual }\end{array}$ & 6 & 14,2 \\
$\begin{array}{c}\text { Cadeira de Rodas } \\
\text { Elétrica }\end{array}$ & 2 & 4,7 \\
\hline Total & 25 & 59,2 \\
\hline
\end{tabular}


E, finalmente, a avaliação a partir do instrumento HAQ, possibilitou esquematizar a classificação quanto ao desempenho ocupacional geral, conforme a Tabela 4.

Tabela 4 - Classificação geral obtida no HAQ

\begin{tabular}{lll}
\hline \multicolumn{2}{l}{ Classificação Geral - HAQ } & \\
\hline Classificação HAQ & Freqüência (n) & $\begin{array}{l}\text { Porcentagem } \\
\text { (\%) }\end{array}$ \\
Com alguma dificuldade & 18 & 42,86 \\
Com muita dificuldade & 14 & 33,33 \\
Sem dificuldade & 10 & 23,81 \\
\hline Total & 42 & 100 \\
\hline
\end{tabular}

\section{DISCUSSÃO}

O presente estudo foi realizado com indivíduos portadores de hemofilia do sexo masculino, assim como Villaça et al. (2001) que referem que os casos em que mulheres são acometidas são raros, já que ambos os cromossomos $\mathrm{X}$ deveriam ser afetados assim. Quanto aos tipos de coagulopatias os resultados são semelhantes às do estudo de Lorenzi et al. (2003) no qual observou-se que a maioria são portadores da hemofilia tipo A (80,95\%), e quanto à classificação, grave em $71,43 \%$ dos casos e o restante moderada.

$\mathrm{O}$ estudo foi realizado com indivíduos entre 18 a 55 anos, sendo que a faixa etária com maior número foi a mais jovem, entre 18 e 25 anos. Assim como no estudo de Garbin et al. (2004), que justificou a menor parcela de pacientes mais velhos provavelmente devido à alta mortalidade ocorrida há alguns anos, com a contaminação dos hemocomponentes pelo HIV e conseqüente transmissão para os hemofílicos que deles faziam uso, o que levou à morte da maioria dos pacientes. Também foi considerado que o tratamento oferecido era bastante restrito e isto poderia ser a causa da alta mortalidade desta população e da baixa expectativa de vida.

No que diz respeito às pessoas que apresentaram co-morbidades associadas (infecção por Hepatite tipo B, Hepatite tipo $\mathrm{C}$ e HIV), percebeu-se, a partir da resposta à pergunta aberta, que muitos também mostraram problemas psicossociais, dificuldades de relacionamentos interpessoais, baixa auto-estima e isolamento social. Alguns justificaram esta realidade devido à dificuldade de aceitação de si mesmos, pelas seqüelas físicas impostas pela doença e, principalmente, quando revelavam ser portadores de alguma doença infecciosa (HIV e Hepatite tipo C) como relatam dois dos indivíduos entrevistados:

"Tenho dificuldade em me aceitar da forma como sou, com as seqüelas que a hemofilia me deixou”.

"A maior dificuldade que sinto é me aceitar como hemofilico e portador de HIV. Já tive vários relacionamentos e essas pessoas me traíram e os meus amigos me abandonaram".

Conforme citado anteriormente (pelo primeiro entrevistado), as seqüelas da hemofilia são decorrentes das manifestações clínicas comuns à patologia, e neste estudo observou-se prevalência das hemartroses e hematomas musculares, com maior comprometimento nas articulações do joelho, cotovelo e tornozelo, o que também foi encontrado em outros estudos, como o de Santos et al. (2007), cuja amostra apresentou $89 \%$ dos acometimentos em regiões articulares, sendo $33 \%$ em joelho, $17 \%$ em cotovelo e $15 \%$ no tornozelo. Os autores também chegaram ao resultado de que o aparelho locomotor é o mais acometido.

A limitação de movimentos dos MMSS e MMII, em geral, pode ser relacionada às dificuldades apresentadas no desempenho das AVD's e AVP's, e aliada a isto há a necessidade do uso de recursos de tecnologia assistiva, principalmente para a higiene (banho, uso do vaso sanitário e higiene pessoal), conforme relatado por um dos entrevistados:

"Eu não consigo fazer muita coisa, andar, me vestir, escovar os dentes, muito das coisas estou fazendo com o braço esquerdo, mas é bem difícil. Acredito que preciso de ajuda, até para sentar no vaso sanitário tenho dificuldade, preciso da cadeira de banho para acoplar no vaso".

Caio et al. (2001) também referem em seu estudo que, ao avaliar a autopercepção do indivíduo com hemofilia a respeito da sua condição, foi possível registrar a interferência da doença na vida diária do seu portador em até $67 \%$ dos indivíduos entrevistados.

Quanto às AVP's, observou-se que a maioria dos indivíduos apresentava limitações, visto que a maioria estava aposentada ou afastada de suas profissões por motivos de doença. Isto contradiz o esperado, que seria a inserção destes indivíduos no mercado de trabalho, demonstrando o quanto a doença interfere em suas vidas, seja em decorrência das seqüelas que impedem o indivíduo de realizar principalmente atividades que exigem esforço físico, seja pelo fato de a pessoa ter que se ausentar muitas vezes do trabalho, devido aos sangramentos que a acometem. Silva et al. (2007) em um estudo de revisão de literatura observaram que o afastamento do trabalho acarreta perdas em todos os sentidos: pessoais, familiares, sociais e econômicas, sendo 
que isso também foi relatado por dois dos entrevistados neste estudo:

"Eu nunca trabalhei, pois "sangrava" muito e não conseguia arrumar emprego".

"A maior dificuldade que sinto é não poder fazer nada, trabalhar, já que sou aposentado por invalidez".

Nesse sentido também, Caio et al. (2001) registraram em uma população de hemofílicos de Campinas seu relato quanto à interferência da doença no trabalho em $80 \%$ dos entrevistados e também na vida escolar de $50 \%$ deles.

Os aspectos psicossociais dos indivíduos portadores de hemofilia são agravados pelo fato da hemofilia ser uma condição quase que, exclusivamente, masculina, e, por razões culturais, a pressão social quanto ao emprego regular tender a ser maior para os homens, vistos, tradicionalmente, como provedores e mantenedores da família, embora essa tendência social venha diminuindo em nossa sociedade.

A classificação obtida no HAQ evidencia que o desempenho ocupacional é prejudicado pelas limitações da patologia, tanto que em sua maioria os entrevistados foram classificados "com alguma dificuldade" e "com muita dificuldade".

Assim, a hemofilia poderá ocasionar incapacidades na esfera física, psicológica e social que vão interferir na autonomia e independência para realizar as atividades do cotidiano, limitar o desempenho nas áreas e contextos de desempenho (AVD's, AVP's, trabalho, lazer) e na mobilidade, que pode ter como conseqüência, também, o comprometimento na auto-estima, na adequação social e na qualidade de vida destes. Desta forma, o tratamento destes indivíduos deve prever as terapias para a reabilitação física e a integração ao tratamento dos aspectos psicossociais.

A Terapia Ocupacional na reabilitação do indivíduo portador de Hemofilia pode ser baseada em princípios da intervenção em ortopedia e traumatologia, que segundo Luzo et al. (2004) objetivam prevenir deformidades, promover independência e autonomia nas AVD's. Dessa maneira, confeccionar órteses e/ou adaptações e estimular as habilidades e potencialidades do indivíduo podem ser estratégias a serem utilizadas. Além disso, a partir deste estudo percebeu-se também a necessidade da atuação do terapeuta ocupacional no âmbito das atividades profissionais, dos relacionamentos interpessoais e do desempenho em contextos sociais.

Os métodos e recursos terapêuticos que podem ser utilizados em terapia ocupacional podem incluir desde o apoio à realização de atividades artesanais, corporais, expressivas, adaptações, até o uso e desenvolvimento de recursos de tecnologia assistiva como órteses, adequação ambiental, além da adaptação da função/ocupação, uso de exercícios ativos, controle de edema, atividades para estimular ganho de força e Amplitude de Movimento (ADM), estratégias de simplificação de tarefas e conservação de energia como também de orientações sobre como realizar as atividades, protegendo as articulações (proteção articular) e por fim, a realização de treino de atividades (LUZO et al., 2004).

O tratamento de Terapia Ocupacional dispensado aos indivíduos portadores de hemofilia poderá ser fundamentado em estimular as potencialidades e habilidades que o indivíduo possui, como também sua criatividade e expressão, minimizando as limitações impostas pela patologia no cotidiano. Outro foco poderia ser prevenir o aparecimento e a progressão de deformidades e incapacidades por meio da manutenção do posicionamento adequado do membro acometido, do controle de edema, da realização de exercícios ativos e passivos, da manutenção da força e ADM muscular e da confecção de órteses e adaptações.

Ademais, o Terapeuta Ocupacional está habilitado para intervir nos aspectos psicossociais através do estabelecimento do programa terapêutico - ocupacional traçado individualmente levando em consideração os papéis desempenhados pelo indivíduo, o contexto em que vive além do significado das atividades para o mesmo. O Terapeuta Ocupacional utiliza-se de vários recursos terapêuticos como forma de promover a motivação, interesses, capacidades e perspectivas, enfocando as capacidades e habilidades do indivíduo promovendo, o restabelecimento de suas habilidades sociais (SANTOS et al., 2007).

Por fim, o terapeuta ocupacional poderá orientar e planejar junto ao indivíduo e aos familiares as estratégias de simplificação de tarefas e conservação de energia além de realizar adaptações ambientais e de utensílios específicos, preocupando-se com as necessidades do paciente. As questões emocionais, sociais e econômicas e ambientais são enfocadas como forma de promover a independência nas atividades de vida diária, de trabalho e de lazer.

Portanto, a Terapia Ocupacional objetiva melhorar o desempenho ocupacional/funcional nas atividades de vida diária, de vida prática, atividades de lazer e na reorganização do cotidiano, estimular a socialização e a auto-estima, garantir a autonomia do indivíduo e sua inserção social; prevenir contraturas e deformidades, auxiliar o paciente a se adaptar ao ambiente (profissional ou residencial), promovendo assim, bem estar e melhora na qualidade de vida tanto do indivíduo quanto de sua família. 


\section{CONCLUSÃO}

Assim, ressalta-se, a importância da abordagem da Terapia Ocupacional como forma de contribuir na reabilitação do portador de hemofilia utilizando recursos terapêuticos para minimizar deformidades e incapacidades, restabelecer função, favorecer independência e autonomia, melhorar os aspectos psicossociais e proporcionar melhora da qualidade de vida. Através da adaptação de função e ambiente, com o uso de recursos de tecnologia assistiva, da orientação de estratégias de facilitação das atividades e da estimulação ao reconhecimento dos próprios indivíduos de suas habilidades, capacidades e potencialidades, é possível superar a visão de que as incapacidades e limitações impostas pela doença sobrepõem-se a estes aspectos e repercutem negativamente na vida dos indivíduos.

Pode-se, então, conhecer as limitações impostas pela patologia no desempenho ocupacional e na participação de indivíduos acometidos pela hemofilia, observando quais são as incapacidades e deformidades decorrentes, como também é possível conhecer os recursos de tecnologia assistiva utilizados e ainda, discutir a atuação do terapeuta ocupacional com esta população.

Por outro lado, há necessidade de realização de outros estudos a fim de destacar a importância da atuação do serviço de terapia ocupacional na assistência ao portador de hemofilia e, dessa forma, obter o apoio institucional do Ministério da Saúde e as condições necessárias para a implantação do serviço nas equipes de saúde. Dessa maneira, seria possível oferecer condições de acesso ao tratamento integral e humanizado, com a participação da família, de modo a prevenir os impactos desta patologia crônica e limitante no desenvolvimento biopsicossocial destas pessoas e de suas famílias.

Percebeu-se a partir deste estudo, que o conhecimento quanto à tecnologia assistiva ainda é restrito a poucos indivíduos e profissionais e, por isso, também seria importante o desenvolvimento de outros estudos para conhecer o impacto do uso destes recursos no cotidiano dos indivíduos estudados, assim como a opinião destes quanto a empregabilidade, durabilidade, estética e qualidade. Ou ainda estudos para poder conhecer o quanto o uso da TA satisfaz os pacientes na superação de suas dificuldades e limitações e o quanto contribui para melhora de sua qualidade de vida e sua inserção social.

Este estudo espera ter contribuído para o enriquecimento do conhecimento da patologia, e primordialmente ter colaborado para a busca de um tratamento integral aos indivíduos portadores de hemofilia.

BARATA-ASSAD, D. A.; ELUI, V. M. C. Limitations in occupational performance of individuals with hemophilia in Hemotherapy Regional Center of Ribeirão Preto, Brazil. Rev. Ter. Ocup. Univ. São Paulo, v. 21, n. 3, p. 198-206, set./dez. 2010.

\begin{abstract}
Hemophilia is a serious hereditary disorder of blood clotting that results in disabilities that influence the performance of affected infants. The assistive technology (AT) is equipment or set of products, modified or purchased or custom made, used to expand the functional performance. This research aimed to identify limitations in occupational performance of the hemophiliacs and the influence of resources on the functionality of the TA. The study was conducted from January to July 2009 at the Regional Blood Center HCFMRP-USP. All individuals with hemophilia followed up were invited. 42 individuals participated through the application of a schedule for the removal of socio-demographic and clinical data, the accessibility barriers and facilitators of the residence and in the community resources used in the TA activities of daily living (ADLs), mobility in resident and community assessment tool and functionality HAQ (Health Assessment Questionnaire). The results show that most respondents have limitations in ADLs, Productive Activities and Work, mobility, and were classified as "with difficulty" in HAQ. The TA resources used by the majority of participants were related to hygiene and use the toilet and assistive devices for ambulation.
\end{abstract}

KEY WORDS: Occupational therapy; Haemophilia A; Self-help devices/utilization.

\title{
REFERÊNCIAS
}

ANTUNES, S. V. Coagulopatias Hereditárias In: PRADO, F. C. Atualização terapêutica. 23a ed. São Paulo: Artes Médicas,
2007

CAIO, V. M.; SILVA, R. B. P; MAGNA, L. A.; RAMALHO, A. 
S. Genética comunitária e hemofilia em uma população brasileira. Cad. Saude. Publica, v. 17, n. 3, p.595-605, 2001.

CARAPEBA, R. A. B. Características epidemiológicas dos portadores de hemofilia no Estado de Mato Grosso. Mato Grosso do Sul, 2006. Dissertação (Mestrado em Saúde Coletiva). Universidade Federal de Mato Grosso. Instituto de Saúde Coletiva.

FERRAZ, M. B.; OLIVEIRA, L. M.; ARAUJO, P. M. P.; ATRA, E.; TUGWELL, P. Crosscultural reliability of the physical ability dimension of the Health Assessment Questionnaire. J Rheumatol., v. 17, n. 6, p. 813-817, 1990.

GARBIN, L. M. Avaliação da qualidade de vida relacionada à saúde em pacientes portadores de hemofilia. Ribeirão Preto, 2004. Trabalho de Conclusão de curso de Especialização em Enfermagem Clínico - Cirúrgica, Modalidade Residência, na Área de Hematologia-Escola de Enfermagem de Ribeirão PretoUniversidade de São Paulo.

LORENZI, T. F., et al. Manual de hematologia-propedêutica e clínica. Rio de Janeiro: Medsi, 2003. p. 513-520.

LUZO, M. C. M.; MELLO, M. A. F.; CAPANEMA, V. M. Recursos tecnológicos em terapia ocupacional - órteses e tecnologia assistiva. In: DE CARLO, M. M. R. P.; LUZO, M. C. M. Terapia Ocupacional - reabilitação física e contextos hospitalares. São Paulo: Roca, 2004. p. 99-127.

LUZO, M. C. M.; LOURENÇÃO, M. I. P.; ELUI, V. M. C. Atuação terapêutico - ocupacional junto a pacientes com comprometimentos traumato-ortopédicos. In: DE CARLO, M. M. R. P.; LUZO, M. C. M. Terapia ocupacional - reabilitação física e contextos hospitalares. São Paulo: Roca, 2004. p.129-152.

SANTOS, E. G.; PORTES, L. L.; SANTANA, A. G.; NETO, E. T. S. Deformidades e incapacidades dos hemofílicos do Centro de Hemoterapia e Hematologia do Espírito Santo, Brasil. Rev. Ter. Ocup. Univ. São Paulo, v. 18, n. 2, p. 86-94, 2007.

SAS Institute Inc. SAS/STAT ${ }^{\circledR}$ User's guide, version 9. Cary, NC: SAS Institute Inc., 2003.

SILVA, S. R.; GUIMARÃES, E. V.; RODRIGUES, A. M. V. N. Aspectos relacionados ao processo de retorno ao trabalho de indivíduos com desordens musculoesqueléticas do membro superior: uma bibliografia comentada. Rev. Ter. Ocup. Univ. São Paulo, v. 18, n. 1, p. 38-43, 2007.

VILLAÇA, P. R.; CARNEIRO, J. D. A. D’AMICO, E. A. Hemofilias In: FALCÃO, R. P.; PASQUINI, R.; ZAGO, M. A. Hematologia: fundamentos e prática. São Paulo: Atheneu, 2001. p.803-818.

WORLD FEDERATION OF HAEMOPHILIA. Global Treatment Centre Directory: Passsport. Canadá, 2006 [Citado em 11 set. 2008]. Disponível em: www.wfh.org.

ZAGO; M. A.; FALCÃO, R. P.; PASQUINI, R. Hemofilias. In: Hematologia: fundamentos e prática. São Paulo: Atheneu, 2001. p. 798-830. 